\section{Die arbeitgeberseitige Kündigung des Chefarztvertrages - Kündigungsgründe und Rechtsschutzmöglichkeiten (1. Teil)}

Wie in allen anderen Arbeitsverhältnissen, kommt es auch zwischen Krankenhaus und Chefarzt immer wieder zu Konflikten, die darin resultieren, dass der Arbeitsvertrag mit dem Chefarzt durch das Krankenhaus gekündigt wird. Der folgende Beitrag beschäftigt sich mit solchen Kündigungen von Chefarztverträgen durch den Arbeitgeber und den Rechtsschutzmöglichkeiten des Chefarztes.

Dieser 1. Teil des Beitrages hat die denkbaren Konstellationen, in denen ein Arbeitsvertrag mit einem Chefarzt durch den Arbeitgeber gekündigt werden kann, zum Gegenstand. In dem 2. Teil, der in der nächsten Ausgabe erscheinen wird, wird auf die Möglichkeiten eingegangen, wie Chefärzte auf bevorstehende oder bereits ausgesprochene Kündigungen reagieren können. Dabei werden Rechtsschutzmöglichkeiten aufgezeigt, aber auch außergerichtliche Schlichtungsmöglichkeiten, wie z. B. eine Mediation, vorgestellt.

\section{Einführung}

Der Chefarzt bekleidet im Krankenhaus eine herausgehobene Stellung. Er ist Leiter einer Fachabteilung und als solcher der Vorgesetzte des ärztlichen und nichtärztlichen Personals seiner Abteilung. Er trägt die Verantwortung für die Patientenversorgung und ist für den Erfolg des Krankenhauses mitverantwortlich. Wie jeder andere Krankenhausarzt auch, steht der Chefarzt in einem abhängigen Arbeitsverhältnis zum Krankenhaus und nicht in einem selbstständigen Dienstverhältnis. Auf das Arbeitsverhältnis mit dem Chefarzt finden deshalb die allgemeinen Regelungen zur Kündigung von Arbeitsverhältnissen Anwendung.

Üblicherweise werden Chefarztverträge auf unbestimmte Zeit geschlossen. Nach Ablauf der meist nicht vertraglich ausgeschlossenen Probezeit gelten für die Kündigung des Vertrages, unbeschadet des Rechts zur außerordentlichen Kündigung aus wichtigem Grund, die vertraglich vereinbarten oder die gesetzlichen Kündi- gungsfristen nach § 622 Bürgerliches Gesetzbuch (BGB). Weil auf den Chefarzt in aller Regel das Kündigungsschutzgesetz (KSchG) Anwendung findet, bedarf jede Kündigung der sozialen Rechtfertigung. Neben betriebsbedingten Kündigungsgründen, die nicht Gegenstand dieses Beitrags sein sollen, können in der Person des Chefarztes oder insbesondere in dessen Verhalten liegende Gründe die Kündigung sozial rechtfertigen. Aus der besonderen Stellung des Chefarztes ergeben sich dabei wiederkehrende Konstellationen, die zur Rechtfertigung der arbeitgeberseitigen Kündigung herangezogen werden.

\section{Regelmäßig kein leitender Angestellter}

Der Chefarzt ist regelmäßig weder im Sinne des KSchG noch im Sinne des Betriebsverfassungsgesetzes (BetrVG) leitender Angestellter.

Nach § 14 Abs. 2 KSchG ist ein Chefarzt leitender Angestellter, soweit er zur selbstständigen Einstellung und Entlassung von Arbeitnehmern berechtigt ist. Die Einstellungsbefugnisse des Chefarztes beschränken sich zumeist auf Vorschlags- oder Mitentscheidungsrechte. Der Chefarzt ist somit nicht selbst zur Einstellung und Entlassung von Arbeitnehmern berechtigt. Sollte eine Prüfung im Einzelfall doch einmal zu dem Ergebnis kommen, dass der Chefarzt leitender Angestellter im Sinne des KSchG ist, kann er gleichwohl gegen eine Kündigung klagen und im Kündigungsschutzprozess obsiegen. In dem Kündigungsschutzprozess kann der Arbeitgeber jedoch ohne Begründung gem. §14 Abs. 2 S. 2 KSchG den Antrag stellen, das Arbeitsverhältnis gegen Zahlung einer Abfindung aufzulösen.

Leitender Angestellter im Sinne des $§ 5$ Abs. 3 BetrVG ist insbesondere, wer zur selbstständigen Einstellung und Entlassung von im Betrieb beschäftigten Arbeitnehmern berechtigt ist (s. o.) und wer regelmä-

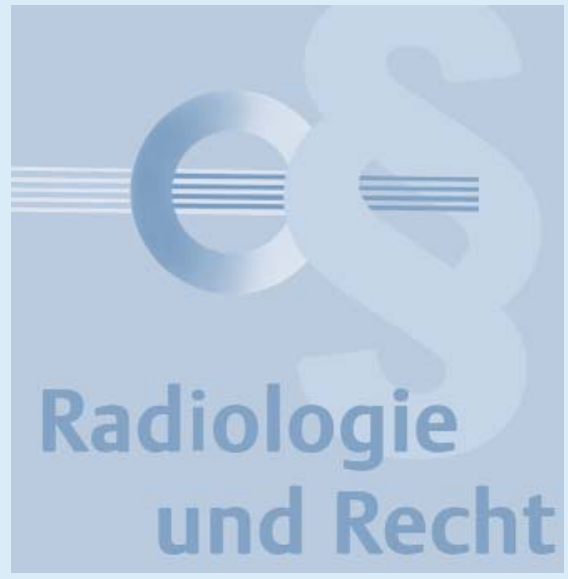

ßig Aufgaben wahrnimmt, die für den Bestand des Unternehmens von Bedeutung sind und deren Erfüllung besondere Erfahrungen und Kenntnisse voraussetzt, wenn er dabei entweder die Entscheidungen im Wesentlichen frei von Weisungen trifft oder sie maßgeblich beeinflusst. Selbst wenn der Chefarzt ausnahmsweise unternehmens- oder betriebsleitende Entscheidungen trifft, soll nach der arbeitsgerichtlichen Rechtsprechung seine Tätigkeit davon regelmäßig nicht geprägt sein. ${ }^{1}$ Üblicherweise findet das BetrVG deshalb auf $\mathrm{Ar}$ beitsverhältnisse mit Chefärzten Anwendung. Vor dem Ausspruch einer Kündigung ist somit in der Regel gem. §102 Abs. 1 BetrVG der Betriebsrat zu hören. Die Anhörung des Betriebsrats ist nur entbehrlich, wenn der Chefarzt ausnahmsweise leitender Angestellter im Sinne des BetrVG ist.

\section{Personenbedingte Kündigungsgründe}

Grund für eine personenbezogene Kündigung kann sein, dass der Arbeitnehmer aufgrund persönlicher Fähigkeiten, Eigenschaften oder nicht vorwerfbarer Einstellungen nicht mehr in der Lage ist, künftig eine vertragsgerechte Leistung zu erbringen. ${ }^{2}$ Die häufigsten Anwendungsfälle der personenbedingten Kündigung des Arbeitsverhältnisses mit einem Chefarzt sind die Krankheit des Chefarztes und der Wegfall seiner medizinischen Eignung. Eine personenbedingte Kündigung ist nur gerechtfertiat. wenn zum Zeitpunkt des Zuaanas

1 vgl. BAG, Beschluss vom 05.05.2010 - 7 ABR $97 / 08$.

2 ErfK/Oetker, § 1 KSchG, Rn. 99 m.w.N. 
der Kündigungserklärung davon auszugehen ist, dass auch nach dem Beendigungstermin des Arbeitsverhältnisses mit weiteren Störungen zu rechnen ist, wenn es nicht fristgerecht gekündigt wird und wenn keine Möglichkeit besteht, den Arbeitnehmer auf einem anderen freien Arbeitsplatz weiter zu beschäftigen, bei dem der Mangel gar nicht oder nur unbedeutend zu Tage tritt. ${ }^{3}$

Das kann beispielsweise der Fall sein, wenn der Chefarzt seine Approbation verliert. Gem. § 2a Bundesärzteordnung (BÄO) darf die Berufsbezeichnung „Ärztin“ oder „Arzt“ nur führen, wer als Arzt approbiert (oder nach den Sonderfällen des $\S 2$ Abs. 2, 3 oder 4 BÄO zur Ausübung des ärztlichen Berufs befugt) ist. Wird die Approbation des Chefarztes während des Arbeitsverhältnisses zurückgenommen oder widerrufen (§5 BÄO), ist der Arzt nicht mehr in der Lage eine vertragsgerechte Leistung zu erbringen. Er darf die vereinbarten ärztlichen Tätigkeiten nicht mehr ausüben. Ähnliche Konstellationen ergeben sich bei anderen Berufen, zu deren Ausübung eine bestimmte Berechtigung erforderlich ist. So können der Verlust der Fahrerlaubnis die Kündigung eines professionellen Kraftfahrers ${ }^{4}$ und der Verlust der Fluglizenz die Kündigung eines Verkehrspiloten ${ }^{5}$ ebenso rechtfertigen, wie der Verlust der kirchlichen Lehrerlaubnis die Kündigung einer Lehrerin ${ }^{6}$. Grundsätzlich fällt es dabei in die Risikosphäre des Arbeitnehmers, ob er die zur Ausübung der vereinbarten Tätigkeit erforderliche Erlaubnis erhält oder nicht. ${ }^{7}$ Die Kündigung eines Arbeitsverhältnisses darf jedoch stets nur als ultima ratio herangezogen werden. Eine Kündigung wegen des Verlustes der Approbation ist deshalb nur möglich, wenn mit einer Wiedererlangung der Approbation in absehbarer Zeit nicht zu rechnen ist und keine Möglichkeit besteht (was bei einem Chefarzt regelmäßig der Fall sein dürfte), den Chefarzt anderweitig im Betrieb zu beschäftigen.

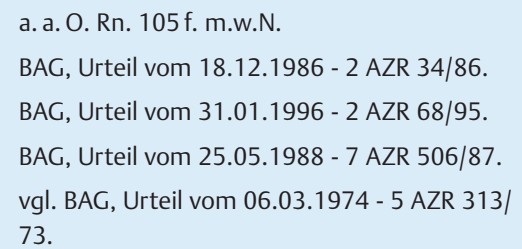

\section{Verhaltensbedingte Kündigungsgründe}

Als Rechtfertigung für die Kündigung eines Chefarztvertrages kommen daneben auch Gründe, die in dem Verhalten des Arztes liegen (vgl. § 1 Abs. 2 S. 1 KSchG), in Betracht.

Eine ordentliche verhaltensbedingte Kündigung eines Chefarztvertrages ist sozial gerechtfertigt, wenn der Chefarzt seine Pflichten aus dem Dienstverhältnis erheblich und in der Regel schuldhaft verletzt hat und mit einer dauerhaften störungsfreien Vertragserfüllung in Zukunft nicht mehr zu rechnen ist. Wegen der ultima-ratioFunktion der Kündigung zur Verhinderung weiterer Störungen im Arbeitsverhältnis ist eine verhaltensbedingte Kündigung nur dann gerechtfertigt, wenn der Arbeitgeber zeitnah vor der Kündigung eine Abmahnung wegen eines gleichgelagerten Verstoßes ausgesprochen hat. Die vorherige Abmahnung des Arbeitnehmers ist lediglich entbehrlich, wenn ein Grund zur außerordentlichen Kündigung vorliegt ( $\$ 626$ Abs. 1 BGB) oder wenn es sich um Verstöße im Vertrauensbereich bzw. um besonders schwerwiegende Verstöße handelt, bei denen der Arbeitnehmer von vorneherein wusste, dass sein Verhalten rechtswidrig ist und vom Arbeitgeber auf keinen Fall hingenommen wird. Eine außerordentliche Kündigung hat innerhalb von zwei Wochen ab Kenntnis des Kündigungsberechtigten von den für die Kündigung maßgebenden Tatsachen zu erfolgen ( $\$ 626$ Abs. 2 BGB). Die für eine außerordentliche Kündigung in Frage kommenden Gründe sind in der Regel geeignet, eine verhaltensbedingte ordentliche Kündigung zu rechtfertigen. Insbesondere ist ein nach §626 Abs. 2 BGB „verfristeter" wichtiger Grund noch geeignet, eine ordentliche Kündigung zu begründen. ${ }^{8}$

In den folgenden Konstellationen kann die verhaltensbedingte Kündigung eines Chefarztvertrages begründet sein:

\section{Behandlungsfehler}

Behandlungsfehler bedeuten eine Verletzung einer Pflicht aus dem Behandlungsvertrag nach §630a Abs. 1 BGB. Sie können

8 ErfK/Oetker, §1 KSchG, Rn. 204. deshalb grundsätzlich die Kündigung jedes angestellten Arztes rechtfertigen.

Ein Chefarzt ist ein gehobener Angestellter mit besonderer Verantwortung. Bei einem solchen Angestellten kann gegebenenfalls sogar bereits ein einmaliges fahrlässiges Verhalten, das geeignet war, einen größeren Schaden herbeizuführen, das zur Fortsetzung des Arbeitsverhältnisses notwendige Vertrauen unheilbar zerstören und einen Grund zur sogar fristlosen Kündigung darstellen. ${ }^{9}$ Wegen der Besonderheit, dass ein einziger auch nur fahrlässiger Fehler des Arztes zum Tod eines Pateinten führen kann, ist es möglich, dass eine solche Situation bei einem Behandlungsfehler eintritt. Zudem kann ein ärztlicher Fehler den Ruf des Krankenhauses beträchtlich schmälern. Auch deshalb kann bereits ein fahrlässiger Fehler die Kündigung des Anstellungsvertrages rechtfertigen. ${ }^{10}$ Unterläuft dem Chefarzt ein sogenannter grober Behandlungsfehler, kann dies die Kündigung des Arbeitsverhältnisses auch ohne vorherige Abmahnung ohne Weiteres rechtfertigen. ${ }^{11}$

Ähnliches wie für Behandlungsfehler dürfte auch für Organisationsfehler gelten. Der Chefarzt ist verpflichtet, durch sachgerechte Organisation und Koordinierung seiner Tätigkeit Behandlungsrisiken, zum Beispiel durch mangelhafte Hygiene, auszuschließen. Als Leiter der Fachabteilung trifft ihn insoweit eine besondere Verantwortung. Unterlaufen ihm in dieser Hinsicht Fehler, können auch diese die Kündigung des Arbeitsverhältnisses rechtfertigen, es sei denn sie liegen auch im Zurechnungsbereich des Krankenhauses.

\section{Falschabrechnung}

Als Nebenpflicht aus dem Arbeitsvertrag ist der Chefarzt zur Rücksichtnahme gegenüber dem Krankenhaus verpflichtet ( $\$ 241$ Abs. 2 BGB). Bei der Abrechnung von Wahlleistungen ist der Chefarzt verpflichtet, sich an die Vorgaben der Gebührenordnung für Ärzte (GOÄ) zu halten. Rechnet der Chefarzt die von ihm erbrachten Wahlleistunaen

9 BAG, Urteil vom 14.10.1965 - 2 AZR 466/64.

10 LAG Düsseldorf, Urteil vom 04.11.2005 - 9 Sa 993/05; BAG, Urteil vom 31.01.1985 - 2 AZR 284/83 (bzgl. Arzthelferin).

11 LAG Düsseldorf, Urteil vom 17.03.1998- 16 Sa 632/96. 
unter Verstoß gegen §4 Abs. 2 GOÄ ab, kann der Eindruck entstehen, in dem Krankenhaus würde nicht korrekt abgerechnet. Das kann sich negativ auf den Ruf des Krankenhauses auswirken. Die Falschabrechnung wahlärztlicher Leistungen durch einen Chefarzt stellt insofern die Verletzung einer vertraglichen Nebenpflicht dar, die einen Grund zur verhaltensbedingten darstellen kann. ${ }^{12}$

Dies gilt auch für den Bereich der vertragsärztlichen Versorgung, falls der Chefarzt über eine persönliche Ermächtigung nach § 116 SGB V, §31a Ärzte-ZV verfügt. Im Bereich der GKV gilt der Grundsatz der „peinlich genauen Abrechnung“ gegenüber der Kassenärztlichen Vereinigung. ${ }^{13}$ Verstößt der Chefarzt gegen dieses Gebot, etwa weil er gegen den Grundsatz der persönlichen Leistungserbringung verstößt, kann dies ebenfalls eine Kündigung des Krankenhauses rechtfertigen. Dies gilt erst recht, weil die Rechtsprechung auch bei Vorliegen einer ansonsten medizinisch ordnungsgemäßen Abrechnung auf einen formalen Schadensbegriff abstellt, weshalb bei einem sogenannten Abrechungsbetrug auch eine Strafbarkeit nach §263 StGB in Betracht kommen kann. ${ }^{14}$ Ein öffentliches Strafverfahren wegen Abrechnungsbetruges gegen den Chefarzt eines Krankenhauses könnte sich erst recht negativ auf den Ruf des Krankenhauses auswirken.

Problematisch sind insbesondere Fälle, in denen die Falschabrechnungen auf einem Fehlverhalten des Chefarztes beruhen, das durch den Krankenhausträger gefördert oder mitverursacht wurde, indem z. B. der zugesagte Personalschlüssel unterschritten wird. Hier kann sogar die umgekehrte Situation eintreten, dass ein Chefarzt nach Abmahnung seinerseits berechtigt ist, das Arbeitsverhältnis außerordentlich zu kündigen, weil ihm der Arbeitgeber kein ausreichendes Personal zur Verfügung stellt. ${ }^{15}$ Nach der Rechtsprechung des Bundesar-

12 LAG Niedersachsen, Urteil vom 17.04.20132 Sa $179 / 12$.

13 LSG Niedersachen-Bremen, Urteil vom 27.04.2004 - 3 KA 209/04 ER.

14 BGH, Beschluss vom 28. September $1994-4$ StR 280/94.

15 LAG Baden-Württemberg, Urteil vom 11.10.2013 - 12 Sa 5/13. beitsgerichts hat ein Arbeitnehmer nämlich einen Anspruch auf eine dem Arbeitsvertrag entsprechende Beschäftigung. Dazu gehört es auch, dass der Arbeitgeber die notwendigen Arbeitsbedingungen (dazu zählen sachliche und personelle Ressourcen) für die Ausübung der vertragsgemäßen Tätigkeit schafft oder erhält. ${ }^{16}$ In Fällen, in denen dem Krankenhaus das fehlerhafte Abrechnungsverhalten bekannt war oder hätte bekannt sein müssen und dieses nicht dagegen eingeschritten ist, kann eine Kündigung unverhältnismäßig sein.

\section{Unterlassene Dokumentation und Codierung}

Üblicherweise ist der Chefarzt aus $§ 630 \mathrm{f}$ BGB und aufgrund seines Arbeitsvertrages verpflichtet, seine Diagnosen und Prozeduren zu dokumentieren und entsprechend den jeweiligen Richtlinien zu codieren. Kommt der Chefarzt diesen Verpflichtungen wiederholt nicht nach und holt er sie auch nicht nach, kann die verhaltensbedingte Kündigung des Arbeitsvertrages begründet sein. ${ }^{17}$

\section{Schlechte Belegungsrate}

Viele Chefarztverträge sehen vor, dass der Chefarzt auf eine ausreichende Belegung hinzuwirken und dafür geeignete Maßnahmen wie kollegiale Kontakte zu niedergelassenen Ärzten, Informationsveranstaltungen für Patienten und ähnliche zu ergreifen hat. Damit wird der Chefarzt verpflichtet, sein Arbeiten auf eine solche Belegungsrate auszurichten. Werden die angestrebten Belegungszahlen nicht erreicht, sind die Hürden für eine Kündigung des Chefarztvertrages zwar hoch. Gelingt es dem Krankenhausträger jedoch nachzuweisen, dass die Ursachen für die schwachen Belegungszahlen in dem Verhalten des Chefarztes liegen, insbesondere in mangelnden Zuweiserkontakten, kann eine Kündigung nach Abmahnung gerechtfertigt sein. ${ }^{18}$

\section{Konkurrenztätigkeit}

Während des rechtlichen Bestehens eines Arbeitsverhältnisses ist einem Arbeitneh-

16 BAG, Urteil vom 10.11.1955 - 2 AZR 591/54.

17 LAG Sachsen, Urteil vom 01.12.2010 - 2 Sa $56 / 10$.

18 LAG Hessen, Urteil vom 21.12.1989 - 12 Sa $568 / 89$. mer grundsätzlich jede Konkurrenztätigkeit zum Nachteil seines Arbeitgebers untersagt. Zum Beispiel kann die Weitergabe von Patientendaten an ein Konkurrenzunternehmen eine verhaltensbedingte Kündigung rechtfertigen. Eine Abmahnung ist in diesem Fall nicht erforderlich, weil der Arzt selbst mit der erstmaligen Hinnahme eines solchen Verhaltens nicht rechnen kann. ${ }^{19}$

\section{Verschweigen einer Straftat}

Hat sich der Chefarzt in seinem Arbeitsvertrag dazu verpflichtet, seinem Arbeitgeber jedes Straf- oder Ermittlungsverfahren und jede Verurteilung mitzuteilen - was regelmäßig der Fall ist -, kann das Verschweigen eine sogar fristlose Kündigung rechtfertigen. Das gilt jedenfalls dann, wenn die Tat in unmittelbarem Zusammenhang mit der Tätigkeit des Chefarztes steht. Wegen der herausragenden Bedeutung eines Chefarztes für ein Krankenhaus kann ein solches Verhalten das Vertrauensverhältnis mit dem Arbeitgeber irreparabel zerstören. ${ }^{20}$

\section{Korruption}

Grundsätzlich sind auch Korruptionsdelikte geeignet, eine Kündigung des Arbeitsverhältnisses zu rechtfertigen.

Dies können im Bereich der sog. Amtsdelikte die Vorteilsannahme und Bestechlichkeit eines Chefarztes nach $\S \S 331,332$ StGB sein. In einem Fall, in dem ein Chefarzt Bestechungsgelder angenommen hatte, um einem bestimmten Bauunternehmer einen Auftrag für einen Klinikbau zu vermitteln, war die Kündigung nur deshalb unwirksam, weil der Krankenhausträger die erforderliche Anhörung der Mitarbeitervertretung ( $\$ 102$ BetrVG) nicht durchgeführt hatte. ${ }^{21}$ Strafrechtlich war dem Chefarzt Bestechlichkeit nach § 299 StGB vorgeworfen worden. Als Chefarzt in einem katholischen Krankenhaus war er kein Amtsträger im Sinne des $§ 11$ StGB. Strafvorschriften, die als Täter einen Amtsträger voraussetzen, konnten deshalb auf den Chefarzt keine Anwendung finden. Das hätte auch gegolten, wenn er Arzt an ei-

19 BAG, Urteil vom 28. Januar 2010 - 2 AZR 1008/08 m.w.N.

20 LAG Hessen, Urteil vom 05.12.2011 - 7 Sa 524/11.

21 ArbG Düsseldorf, Urteil vom 06.04.2011 14 Ca 8029/10. 
nem Krankenhaus in privater Trägerschaft gewesen wäre. Anders hätte es sich allerdings verhalten, wenn er Arzt in einem staatlichen oder kommunalen Krankenhaus gewesen wäre - auch wenn dieses in einer Rechtsform des Privatrechts geführt worden wäre. Ärzte in staatlichen oder kommunalen Krankenhäusern sind nach der Rechtsprechung Amtsträger gem. $\S 11$ Abs. 1 Nr. 2 lit. c) StGB22 und können sich insbesondere auch nach $\S 332$ StGB strafbar machen. Von dieser Vorschrift sind bereits geringwertige Vorteile erfasst, sofern sie nicht der Höflichkeit oder Gefälligkeit entsprechen und sowohl sozial üblich als auch unter Gesichtspunkten des Rechtsschutzes allgemein gebilligt sind. ${ }^{23}$

Alle Angehörigen der Heilberufe, insbesondere also alle Ärzte, gleich wo sie arbeiten, müssen zudem darauf achten, nicht gegen die durch das Gesetz zur Bekämpfung von Korruption im Gesundheitswesen vom 30.05.2016 24 neu eingeführten Strafvorschriften der §§299a ff. StGB zu verstoßen. Diese verbieten es den Angehörigen der Heilberufe, im Zusammenhang mit der Ausübung ihres Berufs einen Vorteil für sich oder einen Dritten als Gegenleistung dafür zu fordern, sich versprechen zu lassen oder anzunehmen, bei der Verordnung von Arznei-, Heil- oder Hilfsmitteln oder von Medizinprodukten, bei dem Bezug von Arzneioder Hilfsmitteln oder von Medizinprodukten, die jeweils zur unmittelbaren Anwendung durch den Heilberufsangehörigen oder einen seiner Berufshelfer bestimmt sind, oder bei der Zuführung von Patienten oder Untersuchungsmaterial einen andern in unlauterer Weise zu bevorzugen. Die Anordnung der Strafbarkeit baut auf den berufsrechtlichen Vorschriften zur Wahrung der ärztlichen Unabhängigkeit bei der Zusammenarbeit mit Dritten in den $\S \S 30 \mathrm{ff}$. MBO-Ä und den sozialrechtlichen Bestimmungen der Unzulässigkeit der Zusammenarbeit zwischen Leistungserbringern und Vertragsärzten nach den $\S \S 73$ Abs. 7, 128 SGB V auf. Ein Verstoß gegen diese Strafvorschriften, der regelmäßig in unmittelbarem Zusam-

22 siehe nur: BGH, Urteil vom 25. Februar 2003 5 StR 363/02; BGH, Urteil vom 19. Oktober 1999 - 1 StR 264/99; Hanseatisches Oberlandesgericht Hamburg, Beschluss vom 14. Januar 2000 - 2 Ws 243/99.

23 Fischer, Strafgesetzbuch, §331 Rn. 25.

BGBI. I, S. 1254. menhang mit der Berufsausübung des Chefarztes stehen wird, kann grundsätzlich geeignet sein, das Vertrauensverhältnis zwischen Chefarzt und Arbeitgeber unwiederbringlich zu zerstören und Grund für eine Kündigung des Arbeitsverhältnisses sein.

\section{Sonderfall Tendenzbetrieb}

Betriebe, die unmittelbar oder überwiegend politischen, koalitionspolitischen, konfessionellen, karitativen, erzieherischen, wissenschaftlichen oder künstlerischen Bestimmungen oder Zwecken der Berichterstattung oder Meinungsäußerung dienen, sind Tendenzbetriebe. ${ }^{25}$ Im Krankenhausbereich sind dies vor allen Dingen Krankenhäuser in kirchlicher Trägerschaft. Rund ein Drittel der Krankenhäuser in Deutschland wird von frei-gemeinnützigen Trägern betrieben. Dies sind zumeist die im Deutschen Caritas-Verband und dem Diakonischen Werk zusammengeschlossenen kirchlichen Träger. ${ }^{26}$

Das verfassungsmäßig garantierte Selbstbestimmungsrecht der Kirche nach Art. 140 Grundgesetz in Verbindung mit Art. 137 Abs. 3 S. 1 der Weimarer Reichsverfassung findet in allen ihr zugeordneten karitativen Einrichtungen Anwendung. Zwar besteht auch in Tendenzbetrieben der Kündigungsschutz des KSchG. Wenn jedoch die von dem Arbeitnehmer eines Tendenzbetriebes erbrachte Arbeitsleistung oder dessen persönliche Eignung dem Tendenzzweck zuwider laufen, kann damit eine arbeitgeberseitige Kündigung begründet werden. ${ }^{27}$ Das kirchliche Selbstbestimmungsrecht verbietet bei der Anwendung des Kündigungs- und Kündigungsschutzrechts die staatliche Fremdbestimmung über Wesen und Auftrag der Kirche. Dem Selbstverständnis der Kirchen ist bei der Güterabwägung zwischen Kirchenautonomie einerseits und Kündigungsschutz andererseits ein besonderes Gewicht beizumessen. ${ }^{28}$

25 ErfK/Oetker, §1 KSchG, Rn. 174 m.w.N. Weth/Thomae/Reichold/Reichold, Arbeitsrecht im Krankenhaus, Teil 4 Rn. 1.

27 ErfK/Oetker, §1 KSchG, Rn. 174 m.w.N.

28 Weth/Thomae/Reichold/Reichold, Arbeitsrecht im Krankenhaus, Teil 4 Rn. 70; BVerfG, Beschluss vom 04. Juni 1985 - 2 BvR 1703/ 83 - 2 BvR 1718/83 - 2 BvR 856/84.
Ob ein Kündigungsgrund wegen eines Verstoßes gegen Wesen und Auftrag der Kirche vorliegt und ob dieser eine ordentliche oder eine außerordentliche Kündigung rechtfertigen kann, wird kirchenrechtlich auch von der Stellung des Arbeitnehmers abhängig gemacht. Loyalitätsverstöße leitend tätiger Mitarbeiter - dazu zählen in diesem Fall auch Chefärzte - wiegen dabei besonders schwer. ${ }^{29}$ Kirchliche Arbeitnehmer können aufgrund vielerlei spezifisch kirchlicher Gründe in Konflikte mit ihrem Arbeitgeber geraten. Dazu zählen unter anderem der Kirchenaustritt ${ }^{30}$, die Nichtbeachtung ethischer Grundsätze bei ärztlicher Tätigkeit und Schwangerschaftsabbruch oder der Verstoß des Chefarztes mit Behandlungsmethoden (,homologe Insemination") gegen Kirchenrecht. ${ }^{31}$ Besondere Beachtung hat zuletzt die Kündigung eines Chefarztes, weil dieser nach der Scheidung von seiner Ehefrau wieder geheiratet hatte, durch ein katholisches Krankenhaus gefunden. ${ }^{32}$ Die Sache liegt derzeit dem Europäischen Gerichtshof zur Entscheidung vor. Grundsätzlich sind Loyalitätsobliegenheiten von Bediensteten eines kirchlichen Krankenhauses gerichtlich nur beschränkt überprüfbar, sodass auch das Verhalten im geschützten privaten Bereich (vgl. Art. 8 Europäische Menschenrechtskonvention), z. B. eine Wiederheirat nach einer Scheidung, die Kündigung eines leitenden kirchlichen Angestellten rechtfertigen kann.

Bei Tendenzkonflikten ist ebenfalls zwischen personen- und verhaltensbedingten Kündigungsgründen zu unterscheiden, weil eine Abmahnung des Arbeitnehmers nur bei verhaltensbedingten Kündigungsgründen für erforderlich gehalten wird.

\section{Fazit}

Weil der Chefarzt bei der Beendigung des Arbeitsverhältnisses grundsätzlich wie jeder andere Beschäftigte des Krankenhauses zu behandeln ist, gelten für ihn die üblichen

29 Weth/Thomae/Reichold/Reichold, Arbeitsrecht im Krankenhaus, Teil 4 Rn. $83 \mathrm{ff}$.

30 BAG, Urteil vom 25. April 2013 - 2 AZR 579/ 12.

31 ErfK/Oetker, § 1 KSchG, Rn. 176 m.w.N.

32 BVerfG, Beschluss vom 22. Oktober 2014-2 BvR 661/12. 
Kündigungsvoraussetzungen. Insbesondere findet auch das KSchG regelmäßig Anwendung. Die arbeitgeberseitige Kündigung eines Chefarztvertrages ist deshalb üblicherweise an den Maßstäben des KSchG zu messen.

Die herausragende Stellung des Chefarztes sowie seine Verantwortung als Leiter einer Fachabteilung verpflichten ihn bei der Ausübung seiner Tätigkeit jedoch zu einer besonderen Sorgfalt. Aus seiner Position ergeben sich typische Kündigungskonstellationen. Sofern er in einem Tendenzbetrieb tätig ist, hat er dessen Wesen und Auftrag noch mehr als andere Angestellte des Betriebes Rechnung zu tragen. Kommt er mit diesen in Konflikt, kann dies - aufgrund seiner herausgehobenen Stellung noch viel eher als bei anderen Angestellten eines Tendenzbetriebes - einen Kündigungsgrund darstellen, der gerichtlich nur sehr eingeschränkt überprüfbar ist.

Der Chefarzt sollte deshalb die Verpflichtungen aus seinem Arbeitsvertrag besonders gut kennen und auf deren Einhaltung achten. Bei Unklarheiten ist ihm dringend zu empfehlen, rechtzeitig das Gespräch mit seinem Arbeitgeber zu suchen und sich um eine Lösung zu bemühen.
Mit Blick auf die weitreichenden Konsequenzen einer Kündigung nicht nur für den Chefarzt, sondern auch für das Krankenhaus, das einen seiner wichtigsten Angestellten verliert, insbesondere aber auch auf die negative Öffentlichkeit eines Kündigungsschutzprozesses, wird bei dem Krankenhaus regelmäßig die Bereitschaft vorhanden sein, den Konflikt doch noch, z. B. im Wege einer Mediation, aus dem Weg zu räumen oder das Arbeitsverhältnis mit einem Aufhebungsvertrag zu beenden. Das liegt auch im Interesse des Chefarztes, weil entweder die Möglichkeit geschaffen wird, das Arbeitsverhältnis doch fortzusetzen, oder mit dem Aufhebungsvertrag eine Abfindung ausgehandelt werden kann und er sich nicht aus einer gekündigten Anstellung bei einem neuen Arbeitgeber bewerben muss.

Kommt es doch zu einer arbeitgeberseitigen Kündigung, streiten jedenfalls die Vorschriften des KSchG für den gekündigten Chefarzt. Weil die Kündigung stets ultima ratio bleiben muss, hat er trotz seiner exponierten Stellung vielfältige Möglichkeiten, sich gegen die Kündigung seines Arbeitsvertrages zur Wehr zu setzen und das Vorliegen der formalen Voraussetzungen der Kündigung sowie ihre Rechtfertigung in Zweifel zu ziehen. Zudem besteht auch nach Erhebung der Kündigungsschutzklage noch die Möglichkeit, den Kündigungsschutzprozess in einem nichtöffentlichen Verfahren vor dem Güterichter zu beenden, in dem alle Methoden der Konfliktbeilegung einschließlich der Mediation Einsatz finden können.

Der Beitrag wird im nächsten Heft fortgesetzt. Er wird sich mit den Möglichkeiten, wie Chefärzte auf bevorstehende oder bereits ausgesprochene Kündigungen reagieren können, und welche Rechtsschutzmöglichkeiten bestehen, um eine Fortsetzung des Arbeitsverhältnisses zu erreichen oder aber dieses einvernehmlich zu beenden, befassen.

Prof. Dr. Peter Wigge

Rechtsanwalt

Fachanwalt für Medizinrecht

Jonas Kaufhold

Rechtsanwalt

Rechtsanwälte Wigge

Scharnhorststraße 40

48151 Münster

Telefon: (0251) $53595-0$

Telefax: (0251) 53595 - 99

E-Mail: kanzlei@ra-wigge.de www.ra-wigge.de 